Décadrages Décadrages

cınéma, à travers champs Cinéma, à travers champs

$7 \mid 2006$

Stephen Dwoskin

\title{
Oblivion de Stephen Dwoskin : la défonce de l'infini
}

\section{François Albera}

\section{(2) OpenEdition}

\section{Journals}

Édition électronique

URL : http://journals.openedition.org/decadrages/435

DOI : $10.4000 /$ decadrages. 435

ISSN : 2297-5977

\section{Éditeur}

Association Décadrages

\section{Édition imprimée}

Date de publication : 10 avril 2006

Pagination : 8-15

ISBN : 978-29700582-3-6

ISSN : 2235-7823

\section{Référence électronique}

François Albera, «Oblivion de Stephen Dwoskin : la défonce de l'infıni », Décadrages [En ligne], 7| 2006, mis en ligne le 04 février 2014, consulté le 02 mai 2019. URL : http://journals.openedition.org/ decadrages/435; DOI : 10.4000/decadrages.435 
1Albert de Routisie, Irène, Paris, L'Or du Temps/Régine Desforges, 1968, (préface de Jean-Jacques Pauvert), p. 35. Les citations que l'on fera ici sont tirées non des dernières éditions du Con d'Irène d'Aragon, que I'on sait maintenant appartenir au grand roman inachevé et en partie détruit, La Défense de l'infini (1928), mais d'une des éditions "clandestines" prudemment titrée du seul prénom de son personnage central et signée Albert de Routisie par décision de l'éditeur devant le refus de l'auteur, alors présumé, de reconnaître pour sien un texte qui l'eût exposé à la justice et à la calomnie.

2 Edition de poche en français - non identifiée à la couleur bienvenue car le narrateur parle de "ce qui pour [lui] se revêtit concrètement d'une apparence, ce mirage de l'eau noire, Irène " venu effacer "l'autre image, la vivante" (id., p. 98).

$\mathbf{3}$ "C'est ainsi que j'envie beaucoup les érotiques, dont l'érotisme est l'expression. Magnifique langage. Ce n'est vraiment pas le mien. [...] Tout ce qui est irrémédiablement pauvre pour les malheureux individus de ma trempe, dans les complications possibles de la volupté, a pour d'autres, je le sais bien, la prodigieuse valeur métaphorique que moi je ne prête qu'aux mots. Je veux dire que les mots me font subir " (id., pp. 34-35). Irène, être de papier, advient de l'écriture et son image - si image il y a - en procède, non l'inverse. Ce n'est pas la perception, ni la réminiscence qui engagent le récit, ce sont les mots: "Comme les mots nous trahissent, je ne voulais pas vous faire croire, en disant votre image, que je vous voyais. Non. Si je vous avais vue! J'essayais parfois désespérément de vous voir, en fermant les yeux, en les ouvrant au contraire très larges sur l'ombre de la pièce. Mais vous étiez là soudainement. Votre démarche. Votre robe. II semblait que vous choisissiez précisément pour venir, le moment qu'à ma table étroite j'écrivais, n'ayant devant
Dwoskin a "adapté» Ma Mère de Georges Bataille - «mixée» avec le Docteur Faustroll d'Alfred Jarry - dans Further and Particular; voici qu'avec Oblivion (2005), il adapterait Le Con d'Irène d'Aragon. Peut-être. Relevons plutôt que dans ce film, le dernier qu'il a réalisé à ce jour, une femme assez austère tient dans ses mains une édition à jaquette noire de ce livre2: qu'elle le lit. Comme Brigitte Bardot dans Le Mépris ou Belmondo dans Pierrot le fou lisaient, dans leur bain, qui le Fritz Lang de Moullet qui L'Histoire de l'art d'Elie Faure.

Mais c'est autre chose, Le Con d'Irène. Et quitte à évoquer Godard, il faudrait plutôt penser à cette scène de Week-end, en contre-jour, où Mireille Darc lit un passage de L'Histoire de l'œil de Bataille à Jean Yanne en ayant l'air de lui raconter sa nuit précédente. D’ailleurs Week-end est aussi un film, le seul de son auteur, où l'on proclame dans les bois des passages des Chants de Maldoror, poème en prose dont est proche par bien des aspects le texte d'Aragon.

Quoi qu'il en soit, Dwoskin lui-même, filmant quelqu'un lisant $L e$ Con d'Irène, l'a-t-il lu? Sans doute. Mais l'a-t-il adapté au cinéma? C'est moins sûr. Disons plutôt qu'il le cite et qu'il est allé y puiser ce qui pouvait l'intéresser sans se préoccuper une seconde d'en «rendre compte», ni d'en respecter la fable ou le sujet - qui est assez particulier dans sa composition et ses régimes d'énonciation.

D'ailleurs ce livre conte l'aventure d'une écriture, il n'est pas de représentation (on peut dire : image, vue, vision, rêve, description), dans Le Con d'Irène, qui ne passe par cette médiation (Le Dit du con d'Irène) ${ }^{3}$.

Pourtant la variation que propose le cinéaste à partir de ce livre éclaire celui-ci ou en éclaire certaines zones d'ombre. Surgissent des personnages qu'on n'avait guère remarqués, demeurant secondaires ou même pis, marginaux. Sauf que ce sont eux qui intéressent le cinéaste et, 
dès lors, l'ensemble du texte est comme orienté différemment, vu sous un autre angle.

Il en va d'un texte comme d'un objet en trois dimensions, à facettes, recoins, arêtes : le mouvement de rotation que le filmage engage en développe des virtualités ou des aperçus qui bouleversent son architecture d'ensemble. Ainsi le narrateur du livre est déplacé sur la figure - de ce fait: une fonction - du voyeur à qui les femmes se présentent, qu'elles provoquent, titillent ou toisent.

«Elles avaient fait une ronde autour de moi, courbées, se passant l'une l'autre le bras autour de la taille pour aller de la main gauche tripoter le bouton de leur voisine, tandis que leurs langues farfouillaient à droite les culs de celles qui se tortillaient pour les toucher. [...] Et les filles me frôlaient de leurs vulves gonflées. Moi, vêtu d'un petit caleçon de toile, je me sentais atteindre un volume mythologique. $\mathbf{4}$

Il s'agit là d'un rêve du narrateur qui, auparavant, a exprimé tout son dégoût pour la petite ville de Lorraine où il réside temporairement chez ses parents $\mathbf{5}$ et dont seule la maison close ("une vraie prison si elle n'avait pas eu sa lanterne») brille pour lui de quelque espérance.

Ce claque, outre les filles qu'on propose à l'usager, comporte des dispositifs de voyeurisme sur lesquels insiste le narrateur: trou de serrure, judas, périscope, fenêtre, poste de guet.

«[...] et elle me fit signe de la suivre. Elle me mena à la porte, regarda par le trou de la serrure $[\ldots]<[\ldots]$ tiens regarde> $[. .$.$] et je pus le voir$ avec une précision incroyable [...] Je cessai de regarder. » 6 «derrière une tapisserie éraillée habilement par une curiosité qui devait être coutumière un petit judas permettait de voir ce qui se passait à côté, sans que le client, trop confiant dans la vertu des Gobelins, pût s'en douter.» $\mathbf{7}$

La vue est ici de celles qu'on trouve chez Raymond Roussel, dans le poème homonyme - où elle procède d'un porte-plume -, ou des descriptions robbe-grilletiennes dans ses premiers romans (parmi lesquels, faut-il le rappeler, il y a un Voyeur):

"J'aperçus d'abord une femme [...]. C'était une pervenche brune avec de tout petits seins [...]. Le bas noir allait à merveille à ses jambes fines et agitées. Elle jouait avec de petites mules rouges qu'elle retirait et remettait sans cesse [...]. [le client] un petit homme trapu [...]. Il avait une grande barbe blonde en éventail. [...] Pour l'instant, le maire, en bannière, étalait sur le lit une alèse pliée. [...] ‘Tu vas voir toi-même. Il a une petite infirmité. [...] > Eh bien non, je n'allais pas voir [...] et m'écartai du poste de guet.»8 moi que le mur. [...] Je savais que dans mon dos, vous alliez et veniez, muette. Parfois vous vous approchiez de moi. Mon cœur battait. Je savais que se retourner c'était vous évanouir. Je ne me retournais pas. J'écrivais." (id., p. 39). Cette dimension scripturale du rapport au réel est exacerbée par la dimension érotique qui, elle aussi, passe par l'écriture et ne se peut épanouir dans la seule vie: "J'ai toujours envié les érotiques, ces gens libres. Ils n'écrivent pas" (id., p. 42); "[...] je m'adonnai donc une fois de plus à ma drogue. J'écrivais du matin au soir. J'évoquais parfois des fantômes. De nouveaux, d'anciens" (id., p. 100). L'émergence du personnage même d'Irène atteste de ce phénomène: "Sur le papier à carreaux qu'on vous donne avec parcimonie, je me pris au jeu de fréquentations nouvelles. Elles étaient assez larges pour charrier dans leurs plis quelques prénoms qui n'éveillaient rien, puis qui revinrent, moins modestement, qui s'éveillèrent. C'est ainsi que chez un roulier qui s'appelait Gentil-Daniel, je fis la connaissance d'Irène. Elle apparut dans la conque d'une période, soudain" (id., p. 45).

$4 / d$. p. 18.

5 On comprend par la suite qu'il s'agit de Nancy (qui est aussi un prénom, celui de Nancy Cunard, amante d'Aragon, liée à ce livre "détruit " quand elle le quitta). Parmi ces aventures de l'écriture, relevons celle-ci: Lorraine/Irène.

$6 / d .$, pp. 23-27.

$7 / d .$, p. 29.

$8 / d$., pp. 29-31. "Etalait sur le lit une alèse pliée". Remarquons cet alexandrin et ses allitérations. II n'en manque pas dans l'ensemble du texte. 
$9 / d .$, p. 83.

10 ld., pp. 51-52. Irène, nous apprend plus loin le texte, est la fille de Victoire, elle-même fille de l'aïeul.

\section{La vue / la vulve}

Plus tard le lecteur, interpellé pour avoir payé «si cher la semaine dernière le droit d'assister au moyen d'un périscope à une scène assez brève que du fond du caveau où on [1]'avait caché, [il] pri[t] pour une exaltation authentique de l'âme humaine [...]" alors que "ce pâle attelage faubourien [...] avait appris par une triste expérience quotidienne l'art de feindre la volupté sans en éprouver la morsure» (id., p. 80), le lecteur est introduit "dans la chambre d'Irène", "Irène qui fait l'amour». Nouvelle scène de voyeurisme, proche de celle de tout à l'heure où la vue se développe ("je la reconnais [...]", "Pour l'homme, il me tourne le dos [...]», «j'aperçois quatre seins qui hésitent à s'abandonner les uns aux autres $[\ldots]$ ", "à moins que [...]»), se fait description de l'étreinte et s'achève sur une promesse du narrateur qui rapportait le spectacle à son lecteur: «je vous permets de jeter un regard sur le con d'Irène. " 9

Ce sont alors les cinq pages les plus fameuses du livre, poème en prose de divers mètres mais en fréquents alexandrins, gros plan qui n'en finit pas de détailler avec une délicatesse de peintre d'estampes japonais ce «lieu", "à l'échelle [des] désirs", que les métaphores de l'infiniment vaste ("sillages des navires", "caravanes dans le lointain des sables», "la couleur de l'été quand on ferme les yeux») exaltent en alternance avec celles de l'infiniment petit («broderie bien partagée par la hache amoureuse ", "hiatus ravissant", "écrin pâle», "dentelles de l'amour»).

Mais ce ne sont pas ces dispositifs de vision et d'écriture qui ont retenu Dwoskin, ni l'intimité, ce «lieu de délice et d'ombre, ce patio d'ardeur", ce "cher abîme vertigineux" d'Irène, c'est un personnage que le lecteur du Con d'Irène a sans doute tendance à oublier au profit du poème qui donne son nom au livre. En effet quand, de la page, naît le personnage d'Irène, quand s'anime une "scène» rurale sous la menace électrique d'un orage imminent ("on dirait, on dirait l'approche de l'amour») - servantes aux pieds nus nettoyant à grande eau les planchers, garçons de ferme à leurs fourches, bêtes que l'on rentre à l'étable, chiens inquiets -, voici: «l'aïeul paralytique»:

«Il veut parler, il veut parler, terriblement parler. [...] Il bave. Il regarde Irène, qui rougit. [...] L'aïeul montre Irène du doigt. Qu'est-ce qu'il a encore ce vieux fou. Ce qu'il doit en penser des inepties. »10

C'est le monologue intérieur de ce vieillard muet et cloué sur sa chaise (qui forme un chapitre seulement) que Dwoskin met au centre de sa «lecture» du Con: ses «remembrances de vieillard idiot». Personnage à la fois repoussant et pitoyable, sur lequel se croisent d'autres personnages - que d'autres films nous ont rendus familiers et qui sont autant de figures du cinéaste "empêché" (Bebindert): "J'avais vingt-cinq ans 


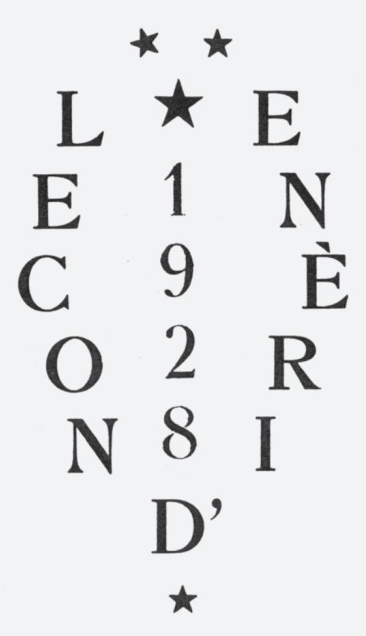

Page de titre de la première édition (1928)

quand je me suis assis pour toujours. »11

$11 / d .$, p. 56.

Il exprime la douleur de la réclusion à laquelle il est condamné, sans espoir de guérison et le «feu [qui] ne s'éteint pas, ne peut pas s'éteindre au cœur de [s] on immobilité» (id., p. 56), ce désir qu'ignore sa femme, ce désir générateur d'images :

«Dans le silence et la quiétude mes yeux dansaient pour émouvoir. Une marée d'images y montait, elle s'interposait peu à peu entre le monde et moi. Corps, corps, corps de tous les gens à la ronde, mes mains clouées vous arrachaient aux vêtements, vous arrachaient les vêtements révélateurs de vos formes damnantes, arrachaient à la fois, écorchaient votre peau tentatrice et laissaient sur vos blancheurs et sur ma cornée de grandes traînées rouges à mourir de la male mort sans confesseur, de la mort divine et grondante qu'appelait sourdement ma chair bouleversée sur la rive impossible à quitter du plaisir, interdit à celui qui n'a plus l'usage de ses mains clouées de part et d'autre des cuisses inertes entre lesquelles dérisoirement se dresse énorme, bonté du ciel suce, branle ou baise! la queue prête à crever les murs, et bandant aux étoiles. »12

Outre les images intérieures, il y a, là aussi, la vue. Le vieillard impotent est un spectateur de toute la vie de la ferme et bien que son «champ 
13 ld., p. 64.

14 ld., p. 66.

Dessin à la plume d'André Masson pour la première édition du Con d'Irène (1928) optique» soit "restreint» («est-ce mon fauteuil qu'on a poussé légèrement?»), il voit. Sa fille Victoire que la mère, consciente ou non de ce qu'elle fait, lui apporte, qu'il voit grandir, devenir femme, et d'autres : «ces désirs mal éteints qu'un rien faisait renaître» sont ravivés par les servantes "dont la seule présence [1] e retournait comme une charrue le sol».

L'une d'elle, «Une grande fille, lente, avec de grandes mains, lentes. [...] Quand il n'y avait personne dans la salle, elle se plantait devant moi sans mot dire. Elle, s'assombrissait. [...] Puis elle écartait bien nettement les cuisses. [...] Un matin au temps des moissons [...]. Brusquement elle releva sa jupe et montra sa motte.»13 Et puis il y a «la recrue des passions entre les commensaux de la ferme» qui se mettent en scène pour le vieillard dans l'embrasure d'une fenêtre ou carrément devant lui.

"J'éprouvais un plaisir positif à voir les hommes et les femmes ensemble. Il me semblait que l'exemple venait à bout de mon infirmité. Cela m'excitait terriblement. Il arrivait même que de tels spectacles m’entraînèrent plus loin que je ne l'eusse pensé.»14

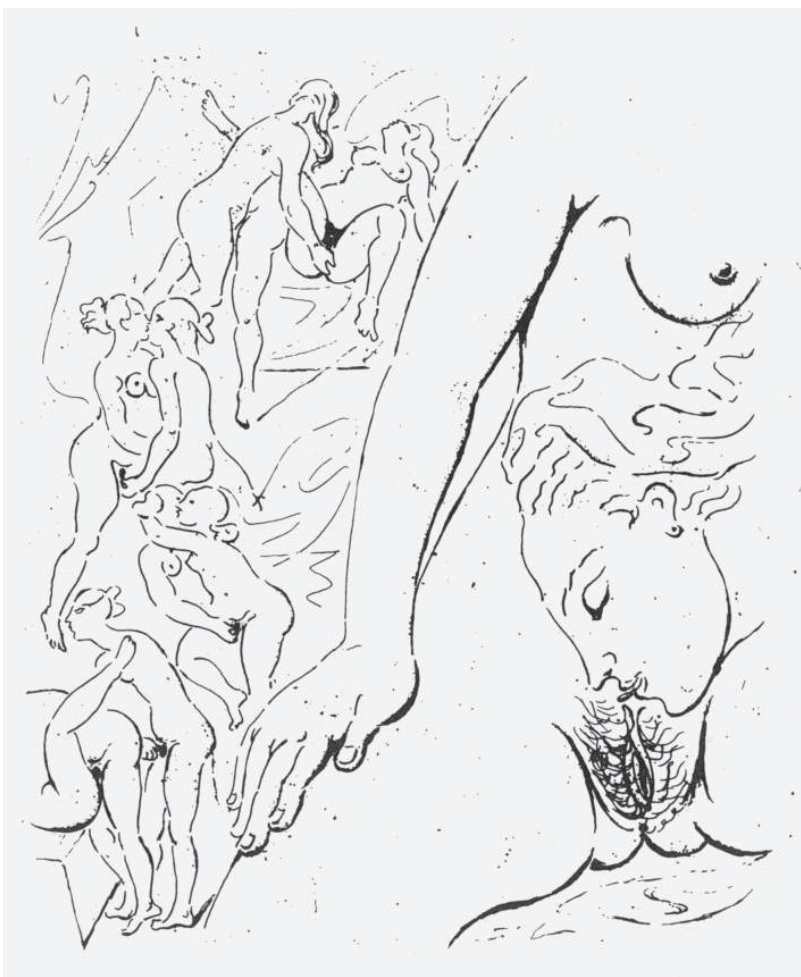


Ce régime monologique est, chez Dwoskin, celui de tout le film; il ne contraste pas, comme dans le roman, avec la parole du narrateur. Celui-ci, on l'a dit plus haut, n'éprouve de volupté que dans les mots, dans l'écriture, il demeure à la fois envieux et distant des "érotiques» qui "n'écrivent pas». L'aïeul muet et paralysé, au contraire, est de ceux-là. Privé de mains il ne peut écrire, mais, privé de mouvement, la volupté qu'il connaît est tout entière liée à la perception et à l'imagination fiévreuse qui la contamine.

Et «rivé au milieu des passions [qui le] mordent sans détruire la digue qui [le] sépare de l'univers» depuis quarante ans, il rit in petto de «la commisération indifférente [qui] entoure le fauteuil des impotents»:

"Imbéciles spectateurs, vous ne comprendrez jamais rien. Je ne donnerais pas ma place pour tout l'or du monde. Soustrait à toutes les considérations puériles des hommes, je consacre ici tout mon temps à la volupté. Mes sens réduits se sont affinés à l'extrême, et c'est dans sa pureté que je connais enfin le plaisir. [...] De temps en temps l'envie violente me ressaisit d'être vivant comme tout le monde. Ce sont des crises brèves, qui me font mieux sentir mon bonheur. »15

Pourtant le propos du film n'a rien de cette superbe, de cette rage, il est, au contraire, empreint de souffrance muette, d'inachèvement et d'inaccomplissement.

\section{Dérobées}

Les premières images du film sont celles, en très gros plan, d'un visage masculin endormi, des yeux clos ou qui battent lentement des paupières. Instauration d'un espace-temps entre l'ensommeillement et le rêve. Une jeune femme entraperçue regarde dans l'entrebâillement d'une porte. Puis une autre qui passe, un corps nu entrevu, aussitôt soustrait au regard. Entre: la restriction du champ de vision pour cause d'immobilité et d'apathie, l'œil se ferait-il protubérant ("cornée [...] aux grandes traînées rouges»), laisse deviner des corps féminins, des regards retournés, des mouvements amorcés aussitôt ramenés en coulisses.

Scandant les scènes, une ampoule nue qui s'allume brutalement dans un claquement et s'éteint de même. Vue en contre-plongée de la position du dormeur tourmenté. Eblouissement, nuit brutale puis lumière étale sur le plafond.

L'enchaînement des situations, des portions de décors, de corps, des ombres projetées, des reflets dans un miroir est, jusqu'au bout du film, légèrement ralenti, et joue continûment sur l'évitement et la soustraction de ce qui est à peine esquissé. Portes dérobées, femmes dérobées. Sept femmes comme il y a la danse des sept voiles autour du corps immobile du dormeur éveillé. 
Il est temps de dire qu'Oblivion (oubli, perte de mémoire) reprend, en un sens, Shadowes from Light, qui était consacré au photographe Bill Brandt. Les modèles féminins mis en scène mettaient en mouvement ses photographies au son du déclencheur, de l'obturateur; celles-ci, usant de courtes focales, déformaient le corps féminin, l'allongeaient, l'incurvaient, le faisaient gonfler ou s'amincir. Là aussi les scènes d'appartement (celui de Brandt peuplé d'objets insolites à la manière surréaliste) étaient discontinues. Des passages, des passantes, des passes. Oblivion reprend ce dispositif et reprend aussi Lost Dreams ou Intoxicated by My Illness où le cinéaste est un homme couché, alité, notamment à l'hôpital, à demi endormi et que frôlent, entourent, croisent les hanches, les bassins et les fesses (son angle de vision) des infirmières. Ici les infirmières sont ces improbables putains venues tourmenter celui qui regarde, rêve et imagine de son lit. Les images lui sont offertes et aussitôt retirées comme dans DynAmo. On pense aussi à Pain Is où il est violenté. La main gantée de limaille scintillante enserre la gorge, une chaussure au talon effilé presse la joue. Il y a tout cela dans Oblivion où les femmes

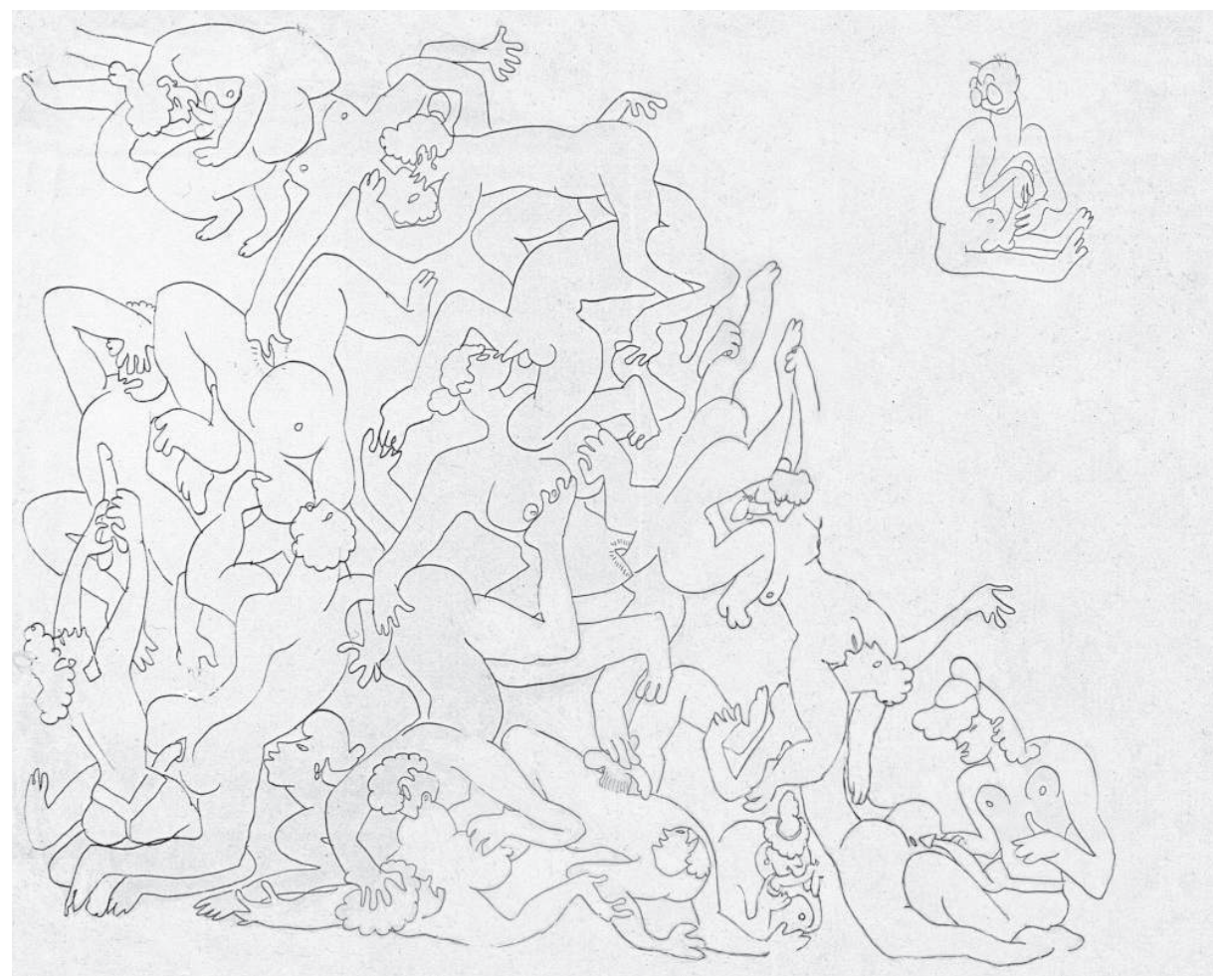


se succèdent au chevet de Dwoskin, penchées sur le spectateur ou lui ouvrant leurs jambes, le moquant aussi ou le maniant, s'exhibant audessus de lui, se rasant, se doigtant, ricanant, se passant la langue sur les lèvres ou serrant celles-ci avec sévérité. Hormis une jeune fille aux grands yeux sombres, apitoyée, inquiète. Est-ce Irène? Et les autres? La mère maquerelle, bouche tordue, cynique, et des filles de bordel trop fardées, au corps fatigué. Cette mascarade évanescente mêle les corps et les gestes: telle caresse commencée dans l'entre-cuisse s'achève dans les cheveux du dormeur que l'on prend d'abord pour une toison, telle plage de peau, tel sillon, anfractuosité, lèvre, replis, muqueuse, glisse d'un visage à un bas-ventre, du nez et de la bouche, de l'œil vertical qui pleure à ses commissures aux lèvres basses. Un objet saille qu'on manipule, un bâton de rouge à lèvres, sur un cintre une combinaison noire se balance. Baisers entre femmes, de celles-ci au dormeur, yeux révulsés, râle muet et le claquement régulier de l'ampoule tel un coup de fouet.

"Si vous avez aimé rien qu'une fois au monde ne me réveillez pas si vous avez aimé!»16

$16 / d .$, p.10.

Le narrateur du Con d'Irène, puisqu'il vit une aventure qui se joue dans les mots, peut exalter son objet des pages durant et laisser Irène à ellemême, qu'il écrit et qu'il admire dans le sommeil ou le rêve de l'écriture, mais l'exercice n'en offre pas moins «la belle image du pessimisme»: l'amant "bande à distance», "il la contemple»: "sur le chott illusoire, une ombre de gazelle...»17 